\title{
Reducing Phase and Amplitude Errors in Restoring Boundary Conditions
}

\author{
David W. Pierce
}

Climate Research Division, Scripps Institution of

Oceanograpy, La Jolla, California 92014-0224

Final Version, February 1996 


\begin{abstract}
Restoring boundary conditions are often used to drive Ocean General Circulation Models. As typically used, such conditions impose time lags and amplitude errors in the seasonal cycle of the model surface tracer fields. Restoring boundary conditions also damp out the high frequency components of the forcing with more damping for higher frequencies; thus models using such conditions systematically underrepresent high-frequency variability in the surface tracer fields. A solution to these problems is presented for use when the forcing field is known beforehand. It is shown that this new formulation significantly reduces the time lags associated with the traditional form of restoring boundary conditions and improves the model's representation of surface variability. The new condition has no run-time overhead and does not impose any additional restrictions on the ability of the model to deviate from observations. The results of using the new boundary condition in an oceanic general circulation model are shown for cases with both monthly and weekly forcing.
\end{abstract}

\title{
1. Introduction
}

Haney (1971) made various physical arguments for forcing Ocean General Circulation Models (OGCMs) with "restoring" boundary conditions:

$$
Q=\kappa\left(T_{A}^{*}-T_{\mathrm{O}}\right)
$$

where $Q$ is the surface heat flux, $\kappa$ is the thermal coupling coeffi cient, $T_{A}^{*}$ is an "apparent atmospheric equilibrium temperature" and $T_{\mathrm{O}}$ is the model's sea surface temperature (SST). $T_{A}^{*}$ can be computed from 
surface heat fluxes and their dependence on temperature, as was done by Han (1984) and Oberhuber (1988) . Marotzke (1994) suggested an alternate approach to calculating $T_{A}^{*}$, which however was a fairly complex procedure. Despite Haney 's emphasis that SST should be restored to $T_{A}^{*}$, many subsequent investigators have nonetheless restored SST to $T_{\mathrm{O}}^{*}$, a historically observed time series of $T_{\mathrm{O}}$. This approach keeps a model from drifting too far from reality but (as shown below) has two distinct disadvantages: 1) it imposes a time lag in the model's surface tracer fi eld; 2) it systematically underrepresents the model's surface variability.

The work presented here is a general method for reducing these problems when restoring to $T_{\mathrm{O}}^{*}$. Physically, it rests on two assumptions: 1) Haney 's parameterization pertains; 2) the effect of internal ocean processes on surface tracer values is smaller than the effect of surface fluxes. If these assumptions are made, then $T_{A}^{*}$ can be derived directly from $T_{\mathrm{O}}^{*}$ and Haney 's method applied. The problems with time lags and underrepresented surface variability are then greatly reduced and the result is a far more realistic simulation of SST.

The second assumption is clearly the weaker of the two and will be discussed in some detail below. However, it must be kept in mind that the improved restoring boundary conditions are an alternative to what will be called "standard" restoring boundary conditions, which assume $T_{A}^{*} \equiv T_{\mathrm{O}}^{*}$. The deleterious consequences of this assumption are shown in detail below and compared to the consequences of the improved restoring boundary condition. The improved condition is an alternative to the standard condition in the sense that it requires no more parameters or information than needed for standard restoring boundary 
conditions. The restoring fi eld $T_{\mathrm{O}}^{*}$ must be known beforehand, but as it is typically based on observations this is a trivial restriction.

This paper is organized as follows. Section 2 shows the derivation of the new restoring boundary condition. Readers more interested in the practical application of the new condition might wish to skip to section 3, which demonstrates the results of using the new condition in an OGCM. The results are discussed in section 4 and conclusions given in section 5 .

\section{Formulation of the boundary condition}

The fi rst step in formulating the new restoring boundary condition is to specify the objective of such a condition: a restoring boundary condition should minimize the difference between the model's tracer field and the observed tracer field. If such an objective is adopted then boundary conditions where the tracer is restored to observations arguably should be rejected out of hand; this is because, as shown below, they impose systematic differences between the model's tracer fi eld and the observed tracer fi eld and thus do not meet the stated objective.

\section{a. Shorter restoring time scales}

The idea that the stated objective can be reached simply by restoring with a short time scale, say 5 days rather than 30 days, perhaps deserves some discussion. There are two problems with this approach: 1) it changes the stability characteristics of the model's thermohaline circulation; 2) it improves the represen- 
tation of SST at the expense of a worse representation of surface heat fluxes and internal heat transport.

To see the fi rst point note that the restoring time constant $r$ is related to $\kappa$ :

$$
\kappa=\frac{\rho_{0} c_{p} \Delta z}{r}
$$

where $\rho_{0}$ is the density and $c_{p}$ the heat capacity of seawater, and $\Delta z$ is the thickness of the OGCM's surface layer. In the limit $r \rightarrow 0$ (to keep the model's surface values close to observations) then $\kappa \rightarrow \infty$. However, the stability of a model's thermohaline circulation is sensitive to $\kappa$ (Zhang et al. 1993 , Power et al. 1994 , Mikolajewicz and Maier-Reimer 1994, Rahmstorf and Willebrand 1996, Pierce et al. 1995 ). Therefore, it is important to choose a value consistent with observations, which suggest that $\kappa$ ranges from about $40 \mathrm{~W} \mathrm{~m}^{-2}$ for small-scale SST anomalies (Oberhuber 1988) to $\sim 2 \mathrm{~W} \mathrm{~m}^{-2}$ for large scale SST anomalies (see Bretherton 1982 ). Seager et al. (1996), considering an average over scales found in an OGCM, suggests using a value $\sim 10 \mathrm{~W} \mathrm{~m}^{-2}$. For a typical $\Delta z$ of $30 \mathrm{~m}$, these values correspond to restoring time scales of 37 days, 2 years, and 150 days, respectively. Using a short restoring time scale such as 5 days, then, is not in accord with observations, and will artifi cially skew the characteristics of a model's thermohaline stability.

The second point devolves out of the observation by Oberhuber (1988) that standard restoring boundary conditions predict zero surface heat flux when a model perfectly reproduces observed SST. Systematic surface heat fluxes (and therefore oceanic meridional heat transports), which are required for an accurate simulation of the climate, are then only possible if model SST deviates from observed SST. Picking the 
correct value of the restoring time scale with standard restoring boundary conditions therefore involves a compromise between accurate SST and accurate surface heat fluxes and meridional heat transport. This is not a problem with the improved restoring boundary condition, which has non-zero surface heat fluxes even if the model exactly reproduces observed SST.

\section{b. Derivation of the improved boundary condition}

Haney (1971) showed that the time evolution of ocean surface temperature $T_{\mathrm{O}}$ can be written

$$
\frac{\partial T_{\mathrm{O}}}{\partial t}=r^{-1}\left(T_{A}^{*}-T_{\mathrm{O}}\right)+\mathcal{I}
$$

where $\mathcal{I}$ represents internal oceanic physical processes that transport heat, such as advection, convection, and diffusion. The problem with applying this equation is that $T_{A}^{*}$ is often not known; rather $T_{\mathrm{O}}^{*}$, a historical time series of $T_{\mathrm{O}}$, is known instead. There are two ways to proceed in this situation: 1) assume $T_{A}^{*} \equiv T_{\mathrm{O}}^{*}$ (which will be called the "standard" approach); or 2 ) assume $\mathcal{I} \ll r^{-1}\left(T_{A}^{*}-T_{\mathrm{O}}\right)$. Note that the applicability of assumption 2 is a function of time scale; seasonal or more rapid changes in surface temperature are strongly influenced by surface fluxes, while internal ocean processes play an important role in multiyear or longer time scales. However as pointed out by England (1992), even over long time scales the detailed density structure of the oceans is sensitive to the seasonal extremes of surface tracers. Therefore a prerequisite for a realistic multi-decade simulation of the ocean is a realistic treatment of surface tracer changes over the short time scales where assumption 2 is valid.

To analyze the implications of these two assumptions, let $T_{\mathrm{O}}^{*}$ be known over a limited time span $\ell$ 
(taken here with no loss of generality to be one year) with $N$ observations in that time period of $T_{\mathrm{O}}^{*}$. Then $T_{\mathrm{O}}^{*}$ can be written as a discrete fourier series:

$$
T_{\mathrm{O}}^{*}=\sum_{n=-N}^{N} c_{n} e^{2 \pi i n t / \ell}
$$

where

$$
c_{n}=\frac{1}{\ell} \int_{0}^{\ell} T_{\mathrm{O}}^{*}(t) e^{-2 i n \pi t / \ell}
$$

Now decompose $T_{A}^{*}$ into fourier components with (to be determined) amplitude and phase shifts relative to $T_{\mathrm{O}}^{*}$ :

$$
T_{A}^{*}=\sum_{n=-N}^{N} w_{n} c_{n} e^{2 \pi i n\left(t+\tau_{n}\right) / \ell},
$$

where $w_{n}$ is the amplitude difference and $\tau_{n}$ the phase difference between $T_{\mathrm{O}}^{*}$ and $T_{A}^{*}$, for the $n$th component.

At this point standard restoring boundary conditions make the assumptions $w_{n} \equiv 1, \tau_{n} \equiv 0$. However an alternative assumption is $\mathcal{I} \ll r^{-1}\left(T_{A}^{*}-T_{\mathrm{O}}\right)$, which allows $w_{n}$ and $\tau_{n}$ to be explicitly calculated as follows. Substituting Eq. 6 into Eq. 3 and rearranging the terms gives

$$
\frac{\partial T_{\mathrm{O}}}{\partial t}+r^{-1} T_{\mathrm{O}}=r^{-1} \sum_{n=-N}^{N} w_{n} c_{n} e^{2 \pi i n\left(t+\tau_{n}\right) / \ell} .
$$

This is simply a linear fi rst-order equation whose solution is:

$$
T_{\mathrm{O}}=e^{-t / r} \int e^{t / r} r^{-1} \sum_{n=-N}^{N} w_{n} c_{n} e^{2 \pi i n\left(t+\tau_{n}\right) / \ell} d t+D e^{-t / r}
$$


where $D$ is an integration constant. The order of the summation and integration can be switched and the integration then performed to give

$$
T_{\mathrm{O}}=\sum_{n=-N}^{N} \frac{w_{n} c_{n} \ell}{2 \pi i n r+\ell} e^{2 \pi i n\left(t+\tau_{n}\right) / \ell}+D e^{-t / r} .
$$

At this point the stated objective is invoked, which means minimizing any differences between $T_{\mathrm{O}}$ and $T_{\mathrm{O}}^{*}$. Therefore equate Eqs. 9 and 4 to give

$$
\sum_{n=-N}^{N} c_{n} e^{2 \pi i n t / \ell}=\sum_{n=-N}^{N} \frac{w_{n} c_{n} \ell}{2 \pi i n r+\ell} e^{2 \pi i n\left(t+\tau_{n}\right) / \ell}+D e^{-t / r}
$$

The $c_{n}$ cancel as does the term $\exp \{2 \pi i n t / \ell\}$. The $D e^{-t / r}$ term goes to zero as time increases, which means the system "forgets" the initial conditions after many time periods $t / r$. Equating the remaining two series term-by-term and rearranging,

$$
\frac{2 \pi i n r+\ell}{\ell w_{n}}=e^{2 \pi i n \tau / \ell}
$$

Rewriting both sides of this equation in the form $a+\mathrm{i} b$,

$$
\frac{1}{w_{n}}+i \frac{\lambda r}{w_{n}}=\cos \lambda \tau+i \sin \lambda \tau
$$

where $\lambda \equiv 2 \pi n / \ell$. Equating the real and imaginary parts of this and taking the ratio gives

$$
\tau=\lambda^{-1} \tan ^{-1}(\lambda r)
$$

A plot of $\lambda \tau$ as a function of $\lambda r$ is shown in Fig. 1. 
The amplitude correction can be calculated from Eq. 11 by noting that the magnitude of the right hand side is unity. Calculating the magnitude of the left hand side and equating it to one yields

$$
w_{n}^{2}=1+(\lambda r)^{2}
$$

A plot of $w_{n}$ as a function of $\lambda r$ is shown in Fig. 2 .

c. Comparison of standard and improved conditions

The calculation of $T_{A}^{*}$ (Eq. 6) given the stated objective and assumption is now complete, using the expression for $\tau_{n}$ in Eq. 13 and for $w_{n}$ in Eq. 14. Standard boundary conditions can then be compared to the improved boundary condition.

Frequently $r=30$ days so for the seasonal cycle $r \lambda<1$. In this case Eq. 13 can be expanded to give

$$
\tau \approx r+O\left((r \lambda)^{2}\right)
$$

This agrees with the intuition that when restoring to observations with a time lag of 30 days, the model should be forced not to the concurrent observed value but to the value approximately 30 days in the future. That way the tracer fi eld will have reached the desired value when the desired time comes about. Note, however, that because of the dependence on $n$ (via $\lambda$ ) in Eq. 13, this correction is different for the different frequency components of the forcing fi eld. Standard restoring boundary conditions assume $\tau_{n} \equiv 0$ for all frequency components, which is a poor assumption when the restoring time scale becomes a substantial fraction of the seasonal timescale, as is often the case. 
The amplitude difference between $T_{\mathrm{O}}^{*}$ and $T_{A}^{*}$ for time-varying components is a function of the component, $n$. For the annual component of the forcing and $r=30$ days the difference is about $13 \%$, i.e. the annual component of the forcing fi eld should be increased $13 \%$ to compensate for the damping effect of restoring boundary conditions. For the semi-annual component $(n=2)$ the difference is about $45 \%$ and as $n$ increases the correction increases rapidly. Standard restoring boundary conditions assume $w_{n} \equiv 1$ for all $n$, which again is a poor assumption. One implication of this is that all variability is reduced, and high-frequency variability reduced preferentially, by using standard restoring boundary conditions.

In practice, constructing $T_{A}^{*}$ from $T_{\mathrm{O}}^{*}$ is straightforward given the existence of a fast fourier transform (FFT) routine. At each latitude, longitude point the forcing fi eld is transformed from the time domain to the frequency domain and each component phase shifted in accordance with Eq. 13 and amplitude corrected in accordance with Eq. 14. Since the amplitude correction increases with $n$, care should be taken not to unrealistically amplify any high-frequency noise in the original data; amplitude correcting only components with frequency lower than an appropriately chosen cutoff is a simple way of doing this. The altered components are then transformed back to the time domain and the sequence repeated until all points have been processed. There is no impact on model running time or effi ciency since all the processing can be done before the actual model run is started. The restoring fi elds must be known beforehand, but as they are typically based on observations this is a trivial restriction.

\section{Results of using the new restoring boundary condition}


Shown here are the results of two sets of Ocean General Circulation Model runs using the new boundary condition. One set of runs was performed using monthly SST values, since this is a widely-used case. The other set used weekly SST fi elds to show the effect of higher-frequency forcing components on the results.

All results were obtained with the GFDL Modular Ocean Model (MOM; Pacanowski et al. 1993 ) however the particular model used makes little difference to the effects of interest here. The model spans $75 \mathrm{~S}$ to $75 \mathrm{~N}$ with a resolution of $1.25^{\circ}$ latitude by $2.5^{\circ}$ longitude and 11 levels in the vertical. Figure 3 shows the model domain. Moderately smoothed bottom topography was used. A 75 minute time step was used at all depths and in both the tracer and stream function equations. The model tracer fi elds were initialized to observed values (Levitus 1982) and the model run for 115 years to obtain the results shown here. This is more than enough time for the surface layers to come to equilibrium with the forcing fi elds, which is the relevant point for this study.

\section{a. Monthly forcing}

In the control case for monthly forcing, standard restoring boundary conditions were used for both temperature and salinity with a restoring time scale of 30 days. Restoring values were taken directly from monthly observed climatology fi elds of sea surface temperature and salinity (da Silva et al. 1995). At each time step the actual values of temperature and salinity were determined by linearly interpolating between the two nearest monthly values. 
In the test case for monthly forcing, the new restoring boundary condition described in section 2 was used for temperature; the boundary condition for salinity was not changed.

Figure 4 demonstrates the time lag that standard restoring boundary conditions impose on the model's response. Plotted are the observed temperature fi eld and model surface temperature in four locations: the North Atlantic (NA), North Pacifi c (NP), South Indian (SI), and the South Atlantic (SA). The locations of the plotted data are shown by the dots in Fig. 3. The data are normalized so the time lag can be more easily seen; to a good approximation the lag is one month, as expected from Eq. 15 with $r=1$ month.

Figure 5 shows the same curves when the new boundary condition is used. In all regions the phase lag has been signifi cantly reduced.

Standard restoring boundary conditions also misrepresent the amplitude of the model's response, as described by Eq. 14. This error is shown for the control case in the upper panel of Fig. 6. Plotted is the amplitude of the model's sea surface temperature (SST) annual cycle divided by the amplitude of the observed SST annual cycle. Regions where the response is within $15 \%$ of the observations are shaded. Standard restoring boundary conditions do a poor job of representing the amplitude of the seasonal cycle; the response is within $15 \%$ of observations in only limited regions. The lower panel shows the same ratio for the new restoring boundary condition. The model's response is much more realistic with large areas of the model now falling within $15 \%$ of observations.

\section{b. Weekly forcing}


In the control case for weekly forcing, standard restoring boundary conditions with a relaxation time scale of 30 days were used for both salinity and temperature. For the test case, the improved boundary condition was used for temperature. Values were restored to weekly SST fi elds taken from NMC data for the year 1992. To avoid spurious amplifi cation of high-frequency noise in the data, amplitude corrections from Eq. 14 were limited to the correction for $n=6$.

Figure 7 shows the annual temperature cycle with weekly forcing at the same four locations used in the previous section. In all locations the improved boundary condition yields a better simulation of SST, in both amplitude and phase. The improved boundary conditions have much more realistic highfrequency detail than do the standard boundary conditions; this is particularly noticeable in the North Atlantic, where the temperature had a double-peaked structure in the summer of 1992. Standard restoring boundary conditions give only the vaguest hint of the early peak; the improved condition captures it in detail. Standard boundary conditions show the late summer peak as being $0.7 \mathrm{C}$ too cold and occurring three weeks later than observed; the improved condition is only $0.15 \mathrm{C}$ too cold and lagged only one week.

An example of $T_{A}^{*}$ calculated from $T_{\mathrm{O}}^{*}$ for this case is shown in Fig. 8 for the North Atlantic location.

\section{Discussion}

The results shown in the previous section demonstrate that the improved restoring boundary condition meet the stated objective of reducing the difference between observed and model-predicted SST, compared to using standard restoring boundary conditions. However it is also worth noting situations where the new 
boundary condition might be less applicable. These arise from the neglect of the internal ocean processes ( $\mathcal{I}$ in Eq. 3) in the derivation. In regions where surface fluxes dominate the surface tracer balance, the neglect is justifi ed and the new boundary condition yields clear improvements. Where the surface fluxes are negligible compared to $\mathcal{I}$, the new boundary condition makes little difference either way. However in regions where the two terms are about equal, increasing the effective surface fluxes as described in Eq. 14 could mistakenly attribute changes in surface tracers to surface fluxes rather than to internal oceanic processes. It should be noted that this is a problem with standard restoring boundary conditions also, rather than a characteristic peculiar to the new boundary condition.

In practice this error is small in medium resolution or coarser models. This can be seen in Fig. 9, which shows the distribution of errors in the amplitude of SST seasonal cycle between the model used in section 3 (which has a resolution of $1.25^{\circ}$ latitude by $2.5^{\circ}$ longitude) and the observations. Were the new boundary condition systematically misattributing to the forcing fi elds changes in the surface tracer that are actually due to internal ocean processes, then it would be expected that the new boundary condition would produce a wider scatter of errors than standard boundary conditions do. However the fi gure shows the converse is true; the new boundary condition has a somewhat smaller standard deviation of errors than the standard boundary condition in addition to reducing the systematic bias towards an overly-small seasonal cycle. Thus there is no evidence that the new boundary condition introduces any systematic errors due to the neglect of the internal ocean processes, at least at a resolution of $1.25^{\circ}$ by $2.5^{\circ}$. A coarser version of the model with $2.5^{\circ}$ by $5.0^{\circ}$ resolution showed the same improvement. Nevertheless it would be prudent 
to check for this kind of error before applying the new condition to an eddy resolving model, especially in regions where advected eddies or "rings" are commonplace, or in a tropical Pacifi c model, where SST in the east is sensitive to regional wind patterns rather than just local fluxes.

It is also useful to point out a situation where the new boundary condition is especially applicable: when both temperature and salinity are restored, but using different time scales. A limiting case of this is when salinity is driven from observed (not diagnosed) net precipitation minus evaporation fluxes. In such cases the effective time lag for temperature and salinity will be different, with the result that the instantaneous values of temperature and salinity will not correctly match up to produce the observed surface density. The improved boundary condition corrects for these different phase lags.

\section{Conclusions}

There are two possible ways of applying Haney (1971) restoring boundary conditions to an OGCM when supplied only with $T_{\mathrm{O}}^{*}$, a historical set of observations of a surface tracer, rather than with $T_{A}^{*}$, the "atmospheric equilibrium" values that Haney 's parameterization requires:

1) Ignore the difference between $T_{\mathrm{O}}^{*}$ and $T_{A}^{*}$. This is the standard approach but it leads to signifi cant time lags in a model's representation of the surface tracer fi eld. It also systematically underrepresents a model's response to time-varying forcing, with greater damping for higher frequencies.

2) Assume that surface fluxes contribute more to surface tracer changes than do internal ocean processes. As shown here, this assumption allows $T_{A}^{*}$ to be computed from $T_{\mathrm{O}}^{*}$, which greatly reduces the 
problems of time lag and reduced variability in the surface tracers. It is also superior to using standard restoring conditions with a very short restoring time scale, as such an approach artifi cially skews the stability characteristics of a model's thermohaline circulation.

The new boundary condition is appropriate for non eddy-resolving models and is of especial benefi $t$ when temperature and salinity are restored with different time scales, as it can compensate for the different time lags the two fi elds would experience with standard restoring boundary conditions. 
Acknowledgments. I would like to thank Tim Barnett of Scripps for his support of this work, and Niklas Schneider of Scripps and Stefan Rahmstorf of the Institut für Meereskunde in Kiel for their insightful comments on the manuscript. The remarks of Bill Holland of NCAR brought the inadequacies of standard restoring boundary conditions to my attention. This work was funded by the DOE under CHAMMP contract DE-FG03-91-ER61215. 


\section{*References}

Bretherton, F. P., 1982: Ocean Climate Modeling . Prog. Oceanog., 11, 93-129.

da Silva, A. M., C. C. Young, and S. Levitus, 1995: Atlas of Surface Marine Data 1994, Volume 1: Algorithms and Procedures. NOAA Atlas NESDIS 6, U.S. Dept. Commerce, 299 pp.

England, M. H., 1992: On the formation of Antarctic Intermediate and Bottom Water in ocean general circulation models. J. Phys. Oceanogr., 22, 918-926.

Han, Y. J., 1984: A numerical world ocean general circulation model. Part II. A baroclinic experiment. Dynamics of Atmospheres and Oceans, 8, 141-172.

Haney, R. L., 1971: Surface thermal boundary conditions for ocean circulation models. J. Phys. Oceanogr., 1, $241-248$.

Levitus, S., 1982: Climatological atlas of the world ocean . Technical report, U.S. Dept. of Commerce, NOAA, 173 pp.

Marotzke, J., 1994: Ocean models in climate problems . In Ocean Processes in Climate Dynamics: Global and Mediterranean Examples, Malanotte-Rizzoli, P. and Robinson, A. R., editors. Kluwer Academic Publishers, 79 109.

Mikolajewicz, U. and E. Maier-Reimer, 1994: Mixed boundary conditions in ocean general circulation models and their influence on the stability of the model's conveyor belt. J. Geophys. Res., 99, 22633-22644.

Oberhuber, J. M., 1988: An atlas based on the COADS dataset: The budgets of heat, buoyancy and turbulent kinetic energy at the surface of the global ocean. Technical report, Max Planck Institut fur Meteorologie, Hamburg, Germany, 199 pp. Technical Report 15. 
Pacanowski, R., K. Dixon, and A. Rosati, 1993: The G.F.D.L. Modular Ocean Model Users Guide . Technical report. GFDL Ocean Group Technical Report No. 2.

Pierce, D. W., K.-Y. Kim, and T. P. Barnett, 1995: Variability of the Thermohaline Circulation in an Ocean General Circulation Model Coupled to an Atmospheric Energy Balance Model. J. Phys. Oceanogr., in press.

Power, S. B., A. M. Moore, D. A. Post, N. R. Smith, and R. Kleeman, 1994: Stability of North Atlantic Deep Water Formation in a Global Ocean General Circulation Model. J. Phys. Oceanogr., 24, 904-916.

Rahmstorf, S. and J. Willebrand, 1996: The role of temperature feedback in stabilising the thermohaline circulation. J. Phys. Oceanog., in press.

Seager, R., Y. Kushnir, and M. A. Cane, 1996: A note on heat flux boundary conditions for ocean models . J. Phys. Oceanogr., submitted.

Zhang, S., R. J. Greatbatch, and C. A. Lin, 1993: A Reexamination of the Polar Halocline Catastrophe and Implications for Coupled Ocean-Atmosphere Modeling. J. Phys. Oceanogr., 23, 287-299. 


\section{Figure Captions}

Figure 1. Normalized time lag $\lambda \tau$ of standard restoring boundary conditions as a function of normalized restoring time $\lambda r$. Also shown are the time lag $\tau$ (months) as a function of restoring time $r$ (months) for the particular case of the annual cycle.

Figure 2. Amplitude error $w_{n}$ of standard restoring boundary conditions as a function of normalized restoring time $\lambda r$. Also shown is the correction as a function of restoring time $r$ (months) for the particular case of the annual cycle.

Figure 3. The domain of the test model. The labeled dots correspond to the analyzed locations. NP= North Pacifi c, NA = North Atlantic, SP = South Pacifi c, SI $=$ South Indian.

Figure 4. Standard restoring boundary condition: Temperature forcing fi eld (solid line) and model SST response (dashed line), with a restoring time of 1 month. The data are normalized so the phase lag can more easily seen.

Figure 5. Improved restoring boundary condition: Temperature forcing fi eld (solid line) and model SST response (dashed line), with a restoring time of 1 month. The data are normalized so the phase lag can more easily seen.

Figure 6. The amplitude of the model's annual cycle divided by the amplitude of the observations. Shaded areas show where the model's amplitude is within $15 \%$ of the observations. Top: standard restoring boundary conditions. Bottom: improved restoring boundary conditions.

Figure 7. Comparison of surface temperature for standard (left column) and improved (right column) 
boundary conditions using weekly forcing. Dashed line: observed SST from 1992. Solid line: model SST.

Figure 8. Model forcing temperature (dotted line) calculated from weekly observed SST (solid line). The observations are from NMC estimated SST fi elds in the North Atlantic during 1992.

Figure 9. Distribution of OGCM model errors in the amplitude of the annual cycle of sea surface temperature. Solid line: standard restoring boundary conditions with restoring to observed values. Dashed line: new boundary condition. 


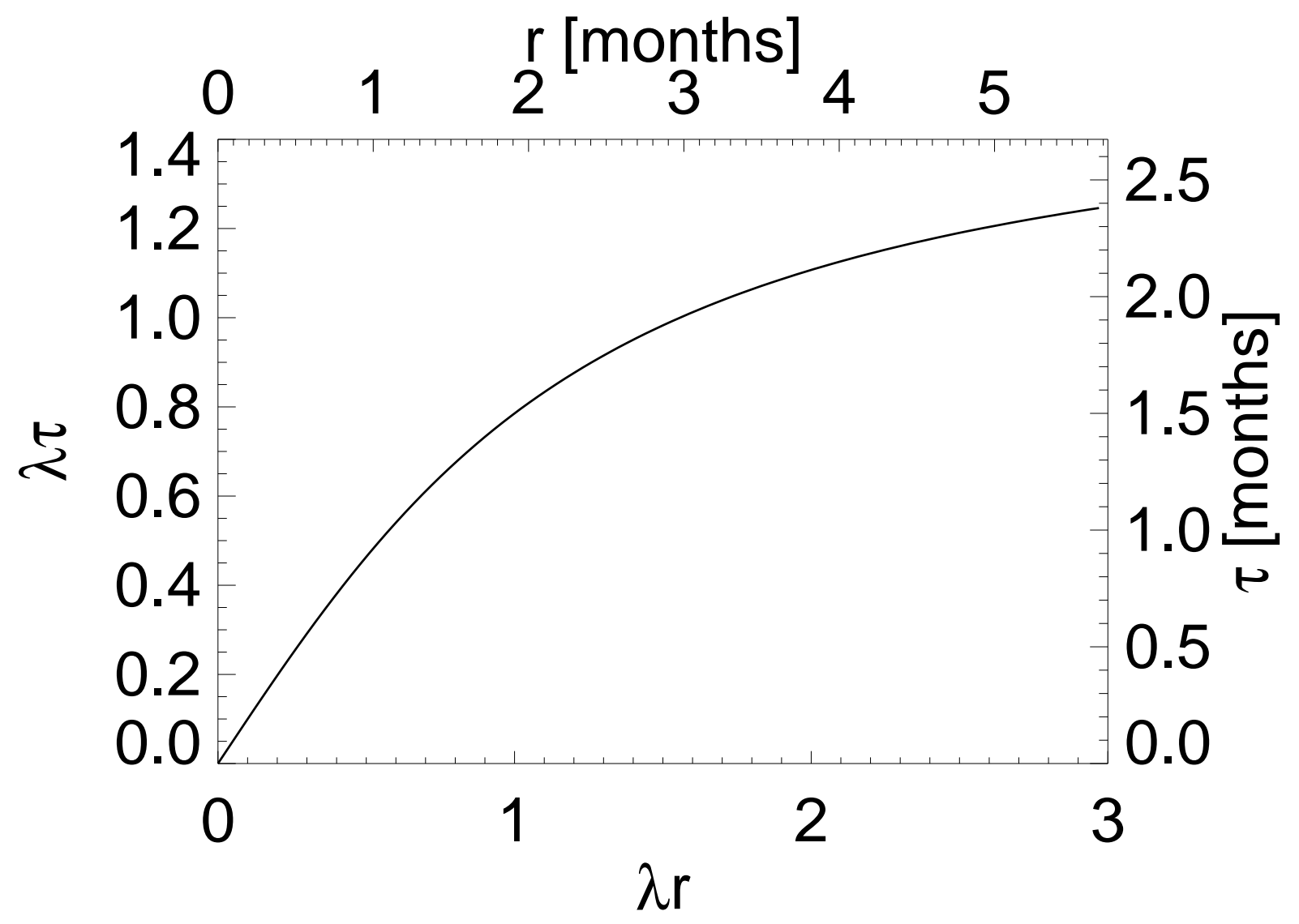

Figure 1: 


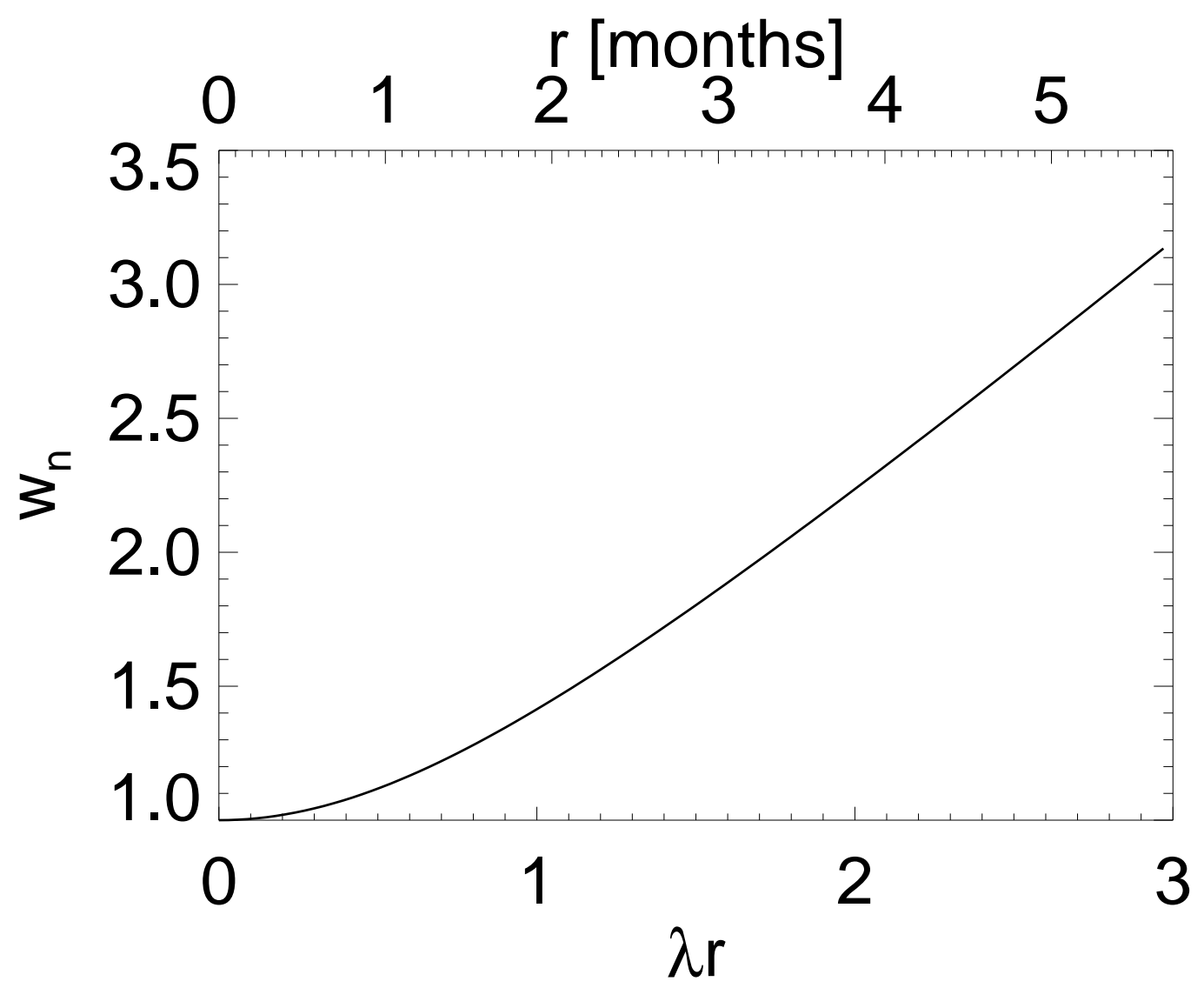

Figure 2: 


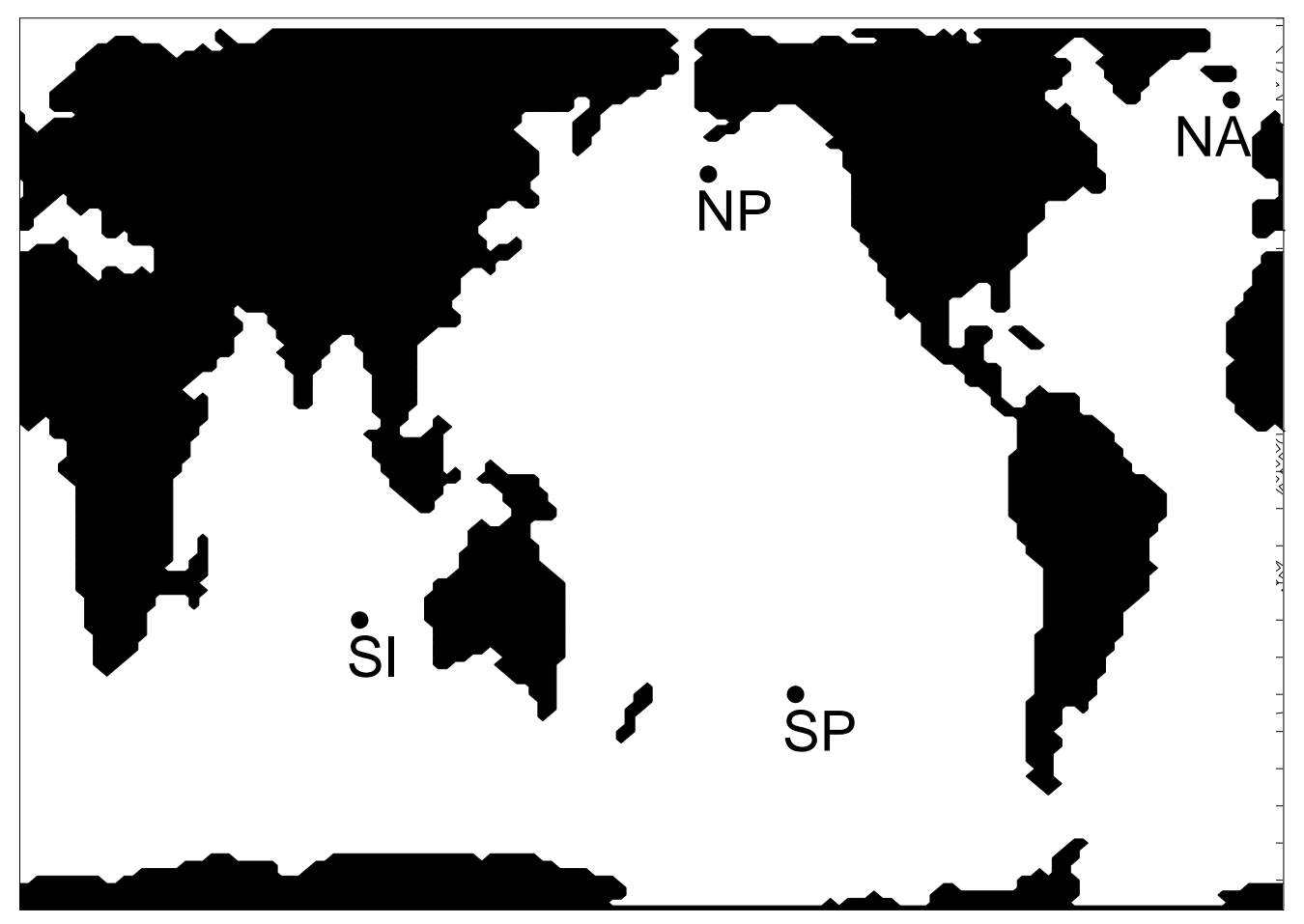

Figure 3: 

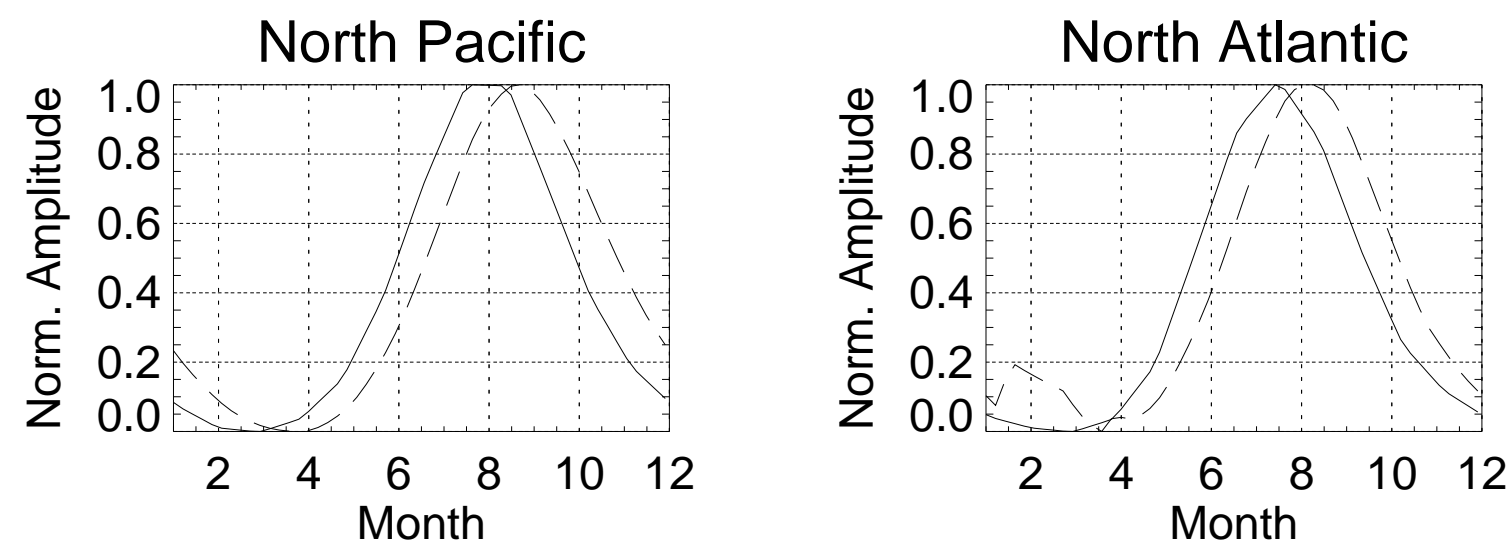

South Pacific

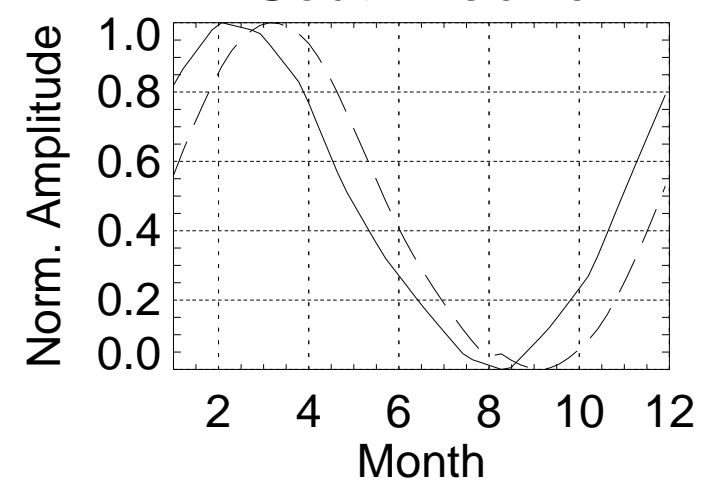

South Indian

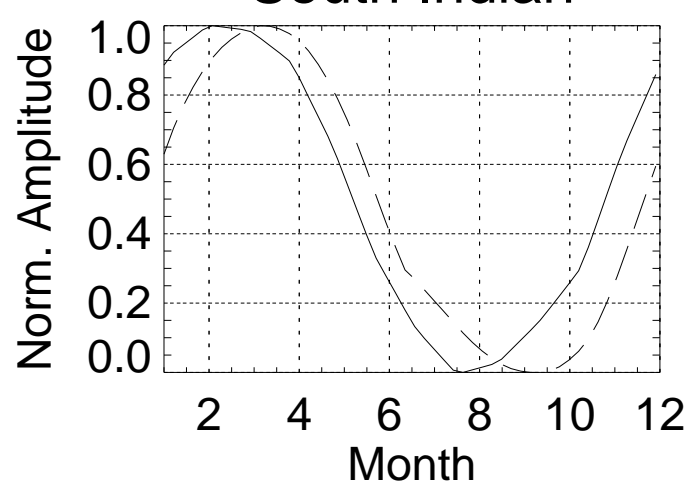

Figure 4: 

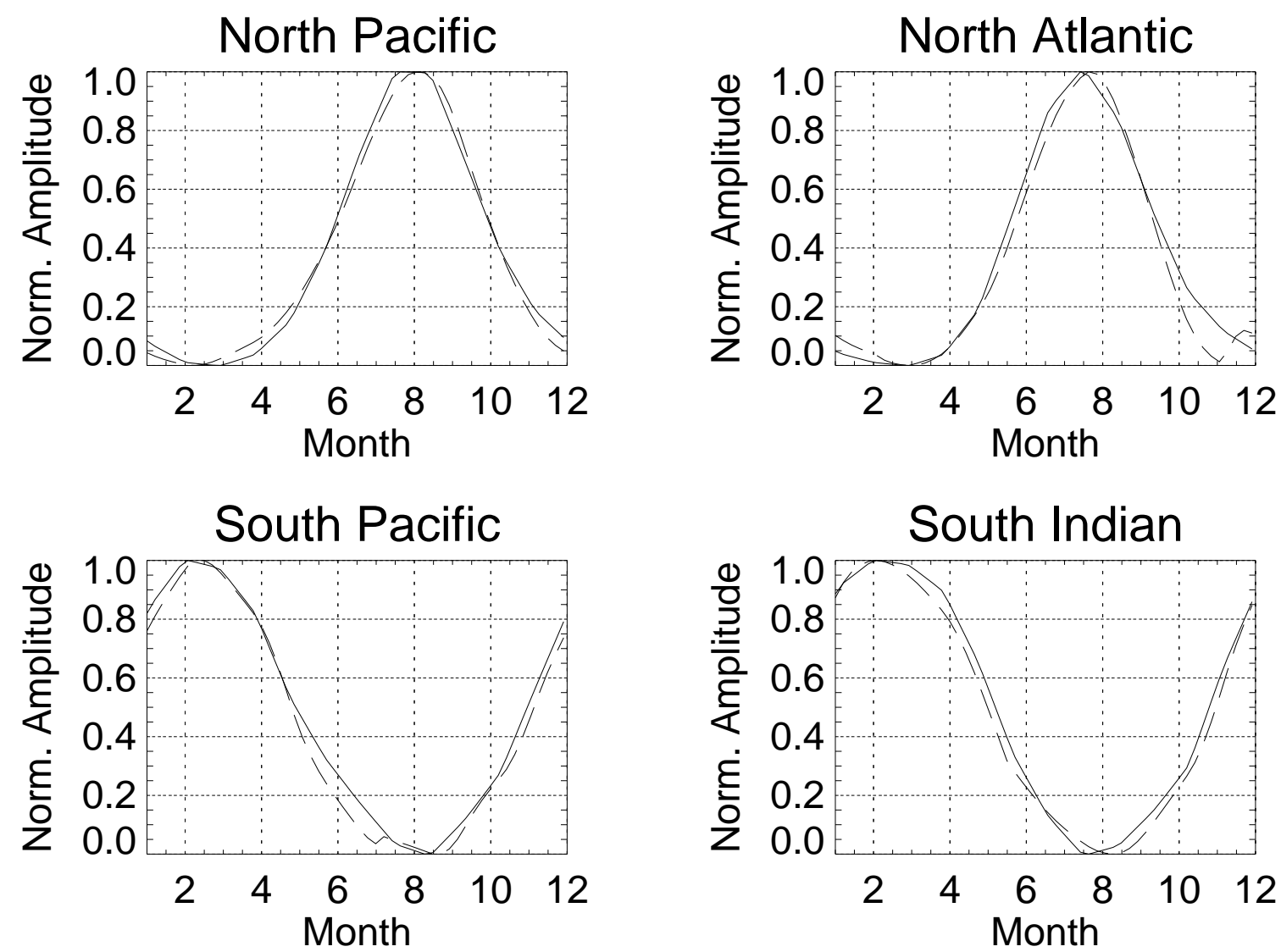

Figure 5: 


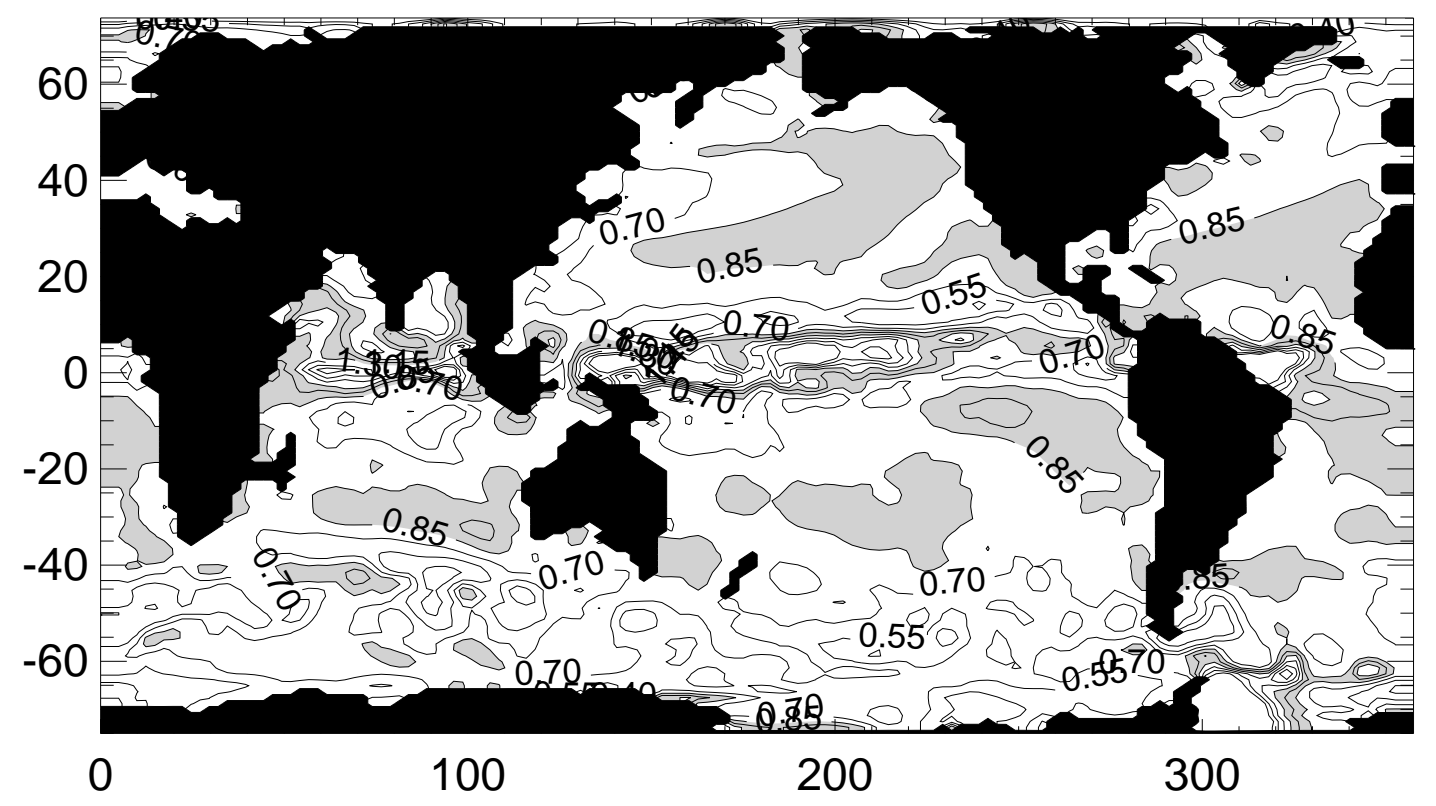

Contour Interval: 0.150

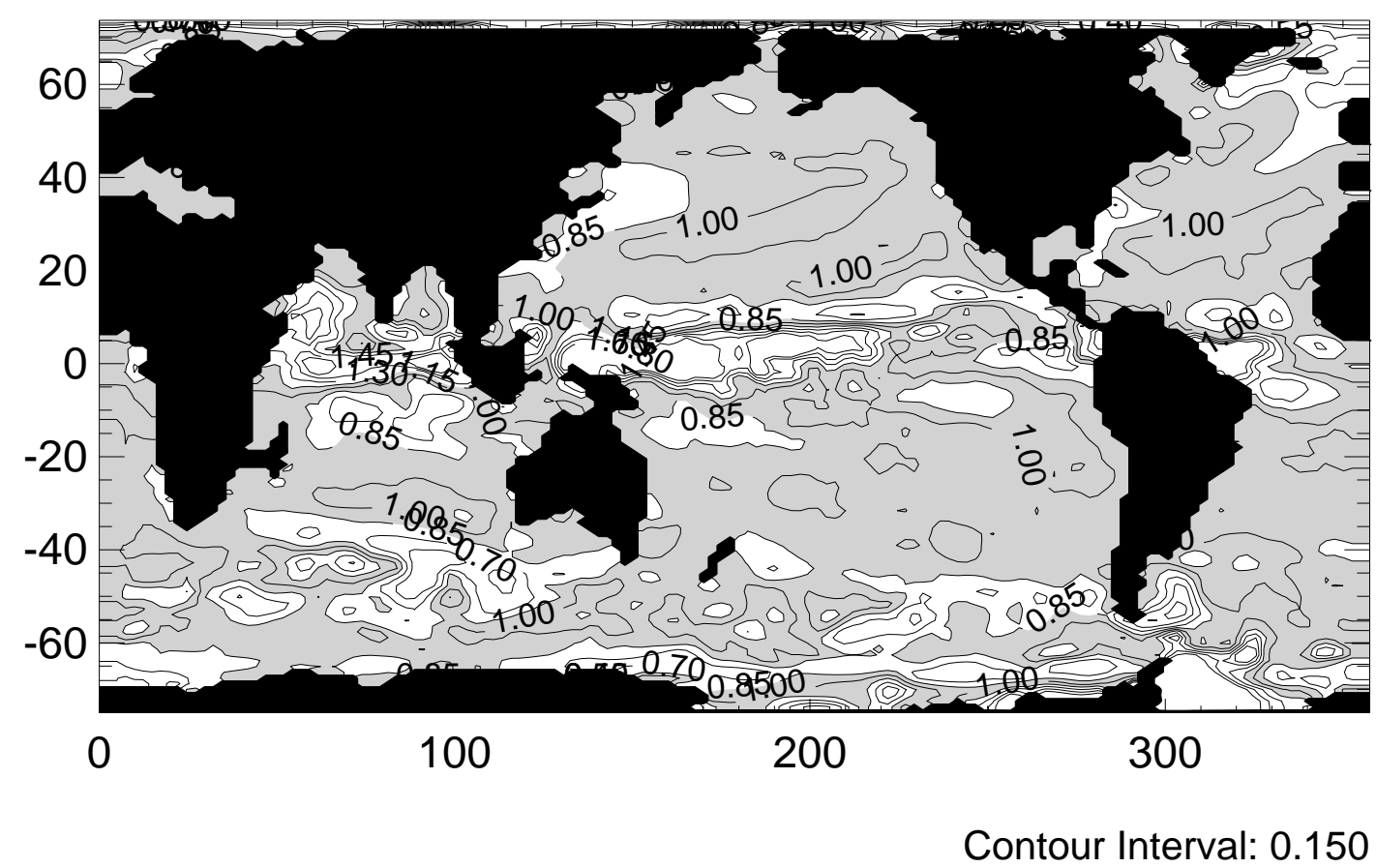

Figure 6: 


\section{Standard}
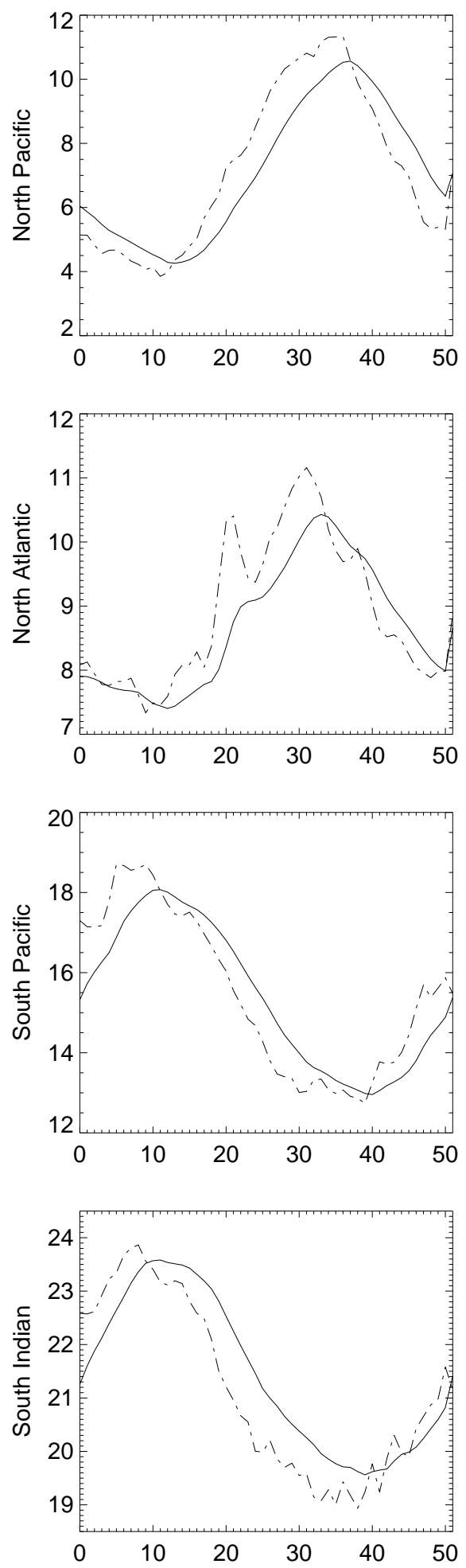

Improved
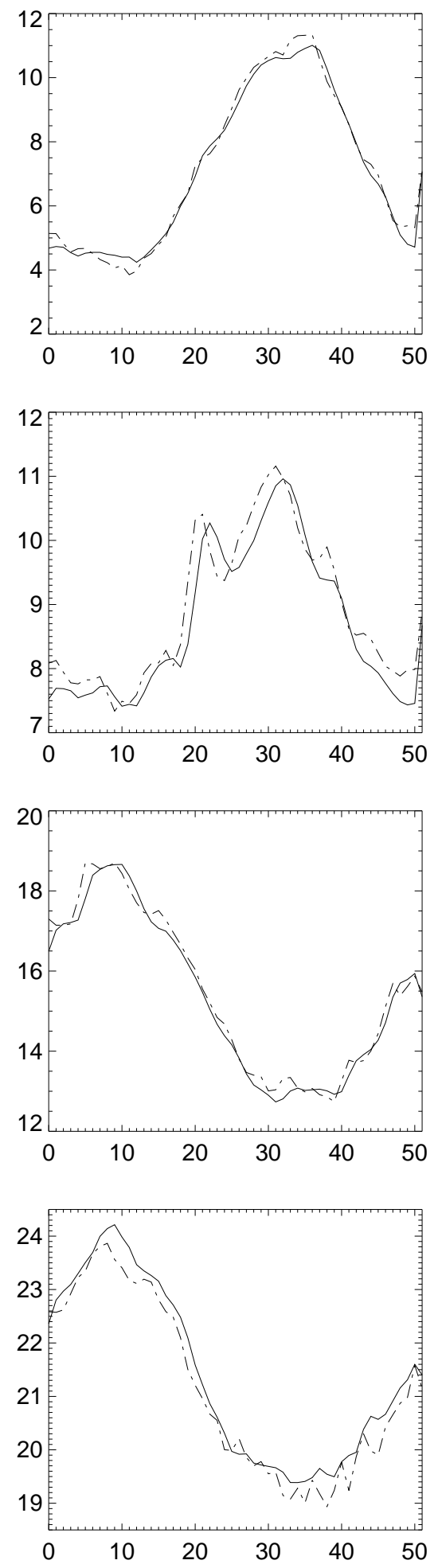

Figure 7: 


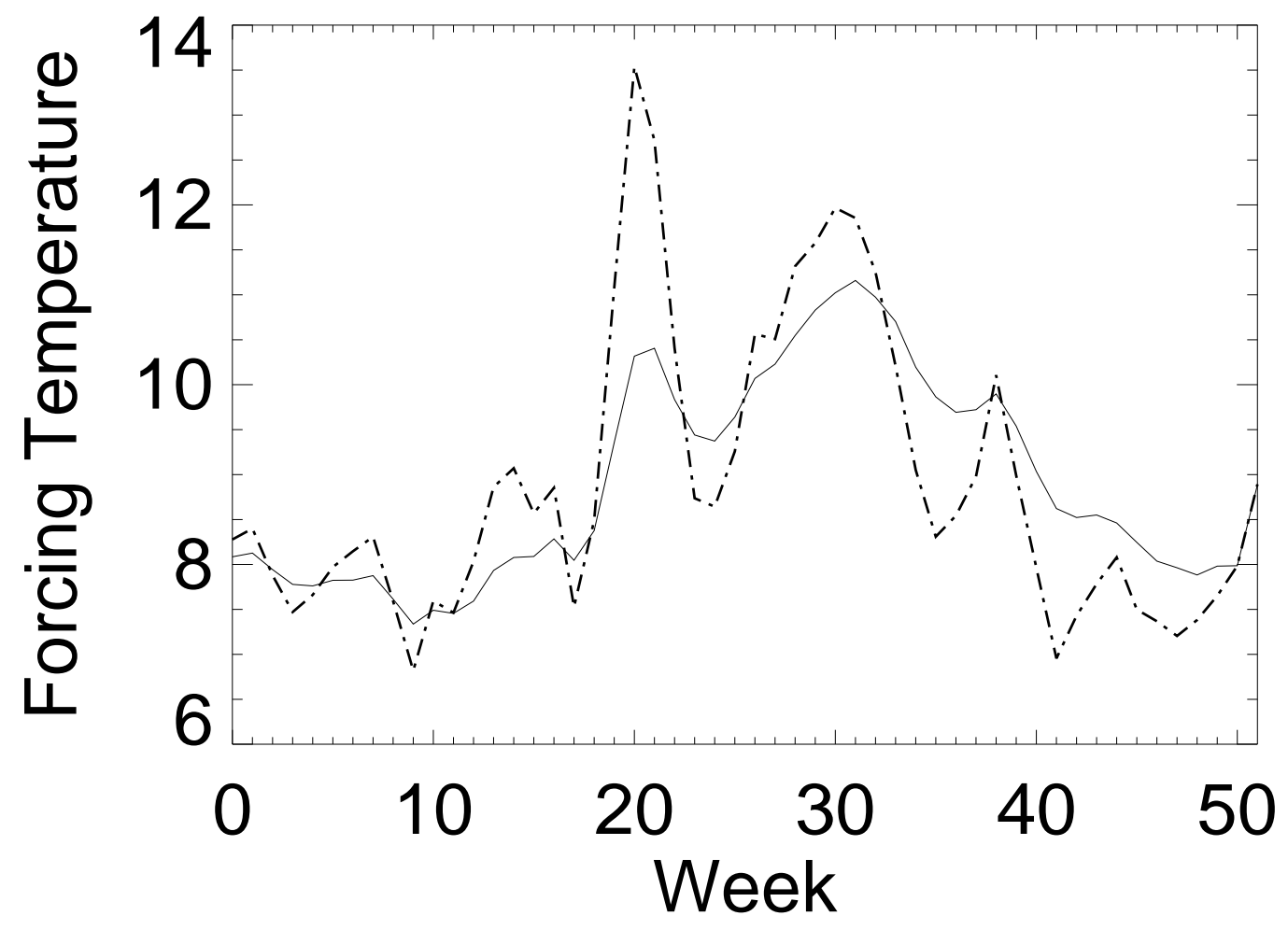

Figure 8: 


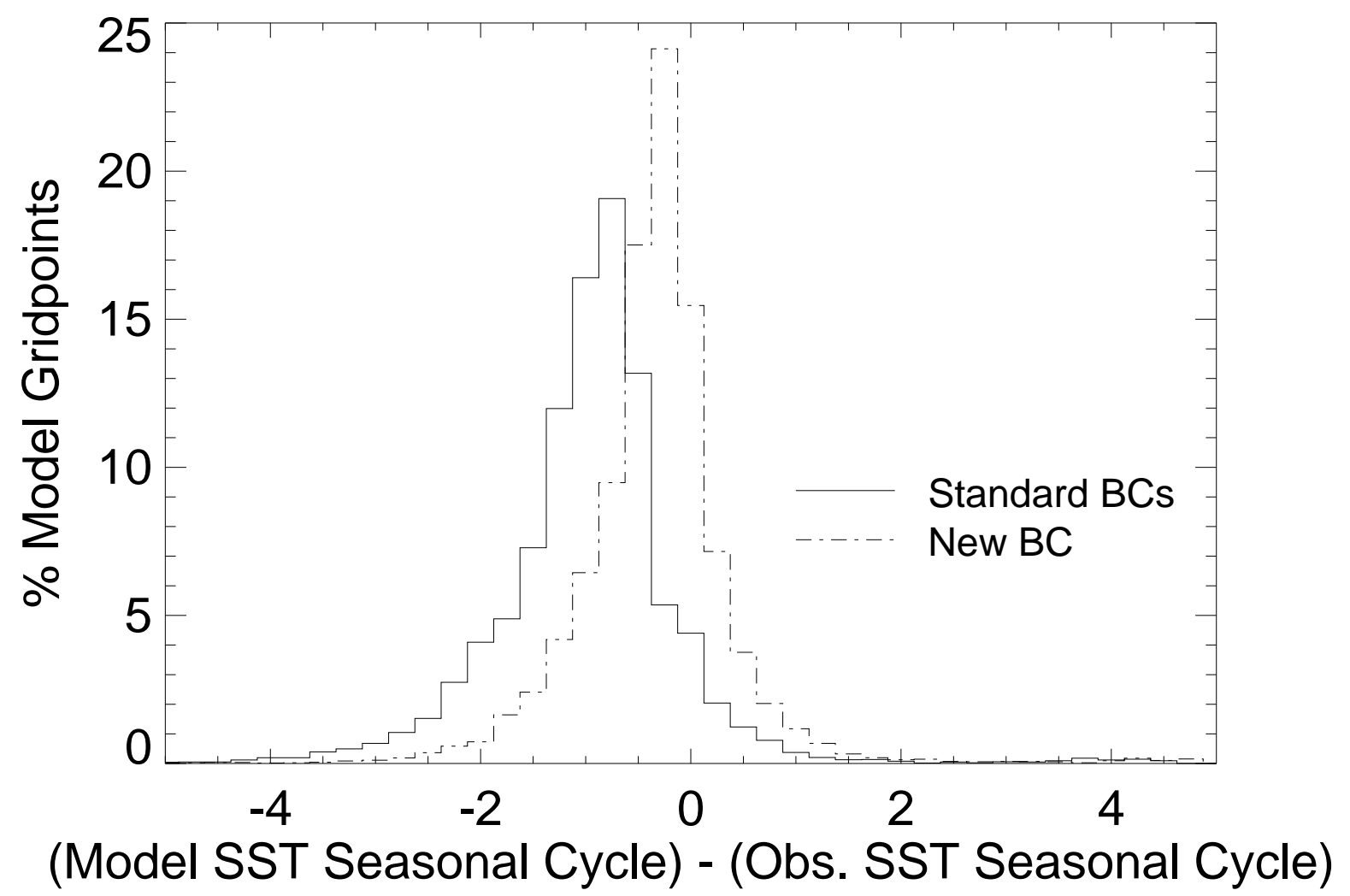

Figure 9: 